Molecules 2005, 10, 971-977

molecules

ISSN 1420-3049

http://www.mdpi.org

\title{
Terpenoids from Cleome droserifolia (Forssk.) Del.
}

\author{
Hesham I. El-Askary \\ Pharmacognosy Department, Faculty of Pharmacy, Cairo University, 11562 Cairo, Egypt; e-mail \\ heshamaskary@yahoo.com
}

Received: 23 January 2005; in revised form: 15 April 2005 / Accepted: 17 April 2005 /

Published: 31 August 2005

\begin{abstract}
A new diacetyl triterpene lactone, drosericarpone (2), was isolated from the hexane extract of the herb Cleome droserifolia, together with buchariol (1, a sesquiterpene oxide, isolated for the first time from Cleome species) and stigmasterol glucoside (3). The structures of 1-3 were identified by spectroscopic means.
\end{abstract}

Keywords: Cleome droserifolia, Cleomaceae, buchariol, drosericarpone

\section{INTRODUCTION}

Cleome droserifolia (Forssk.) Del. belongs to Family Cleomaceae [1,2]. Cleome species are generally used in folk medicine as stomachics, rubefacients and in the treatment of scabies, rheumatic fever and inflammation [3-6]. The dried herb of C. droserifolia, locally known as Samwah, Afein, Reeh-El-Bard [7], is used by herbalists in Egypt as a hypoglycemic agent, and its decoction is widely used by the Bedouins of the southern Sinai for the treatment of diabetes [8]. Several studies were carried out to confirm the hypoglycemic effect of the decoction of this herb [8-10].

Flavonoids [7,11-15] and sesquiterpenes (carotol and an unidentified one) were isolated from $C$. droserifolia [22]. To date, several dammarane triterpenes have been isolated from genus Cleome, viz. C. amblyocarpa Barr. Et Murb. [16] (syn. C. arabica auct. Non L. and C. africana Botsch [17,18] and C. brachycarpa Vahl ex. DC (Punwar) [19-21], but nothing has been reported on the isolation of dammarane triterpenes from Cleome droserifolia. Therefore, the following study was carried out to isolate and identify the constituents of the hexane extract of the plant. 


\section{Results and Discussion:}

The hexane fraction of the ethanolic extract of the powdered herb of $C$. droserifolia afforded three terpenoidal compounds 1-3. The identification of these compounds was accomplished by examination of their spectral data $\left({ }^{1} \mathrm{H}-,{ }^{13} \mathrm{C}-\mathrm{NMR}, \mathrm{COSY}, \mathrm{HMQC}, \mathrm{HMBC}\right.$ and EIMS) and supported by comparison with published data of related compounds [16-30].

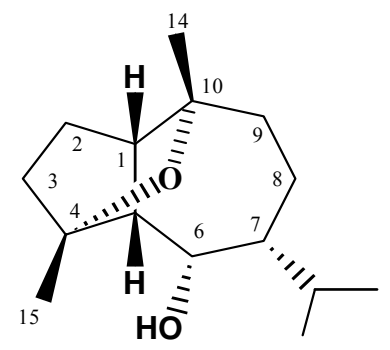

Compound 1

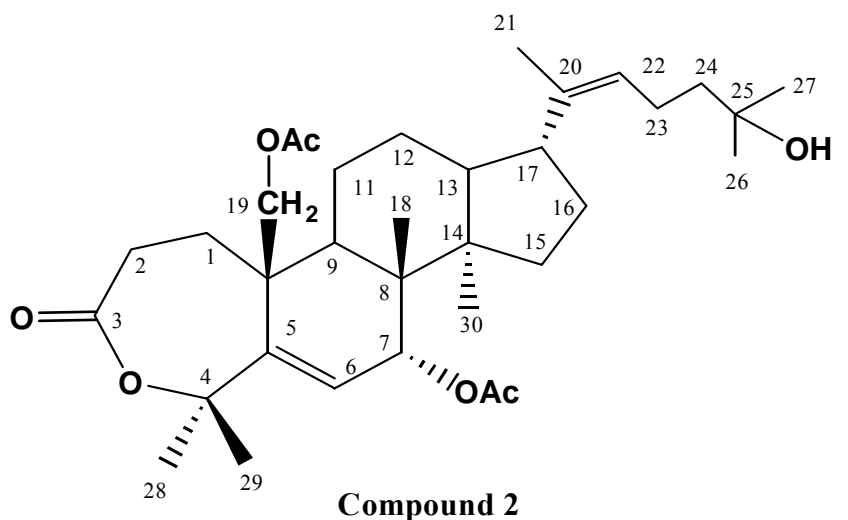

Compound 2

Compound 1, $\mathrm{C}_{15} \mathrm{H}_{26} \mathrm{O}_{2}$, EIMS, $m / z 238[\mathrm{M}]^{+}$, was identified as buchariol, previously isolated from the herb Salvia bucharica, by comparing its spectral data (Table 1) with that reported for this compound [24]. The complete assignment of the ${ }^{13} \mathrm{C}-\mathrm{NMR}$ data of $\mathbf{1}$ was accomplished using 2D NMR spectra (HMQC and HMBC) and is reported here for the first time (Table 1 and Figure 1).

Figure 1. Long range correlations observed in the HMBC spectra of $\mathbf{1}$ and $\mathbf{2}$

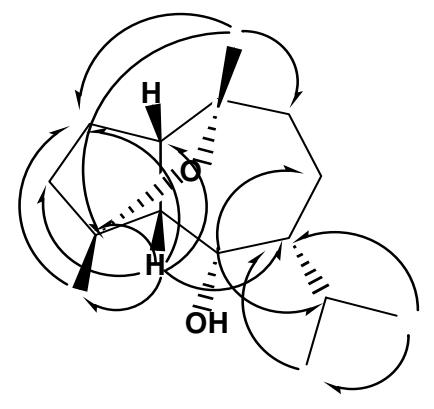

1

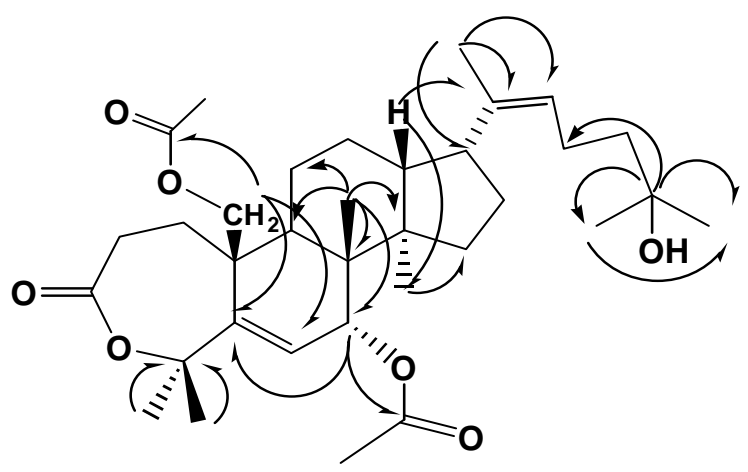

2

Compound 2 was isolated as an oily residue and had a molecular formula of $\mathrm{C}_{34} \mathrm{H}_{52} \mathrm{O}_{7}\left({ }^{13} \mathrm{C}-\mathrm{NMR}\right.$ data and DEPT experiment). Analysis of the ${ }^{1} \mathrm{H}$ - and ${ }^{13} \mathrm{C}$ NMR spectra of 2 with the aid of 2D-NMR spectra $\left({ }^{1} \mathrm{H}-{ }^{1} \mathrm{H}\right.$ COSY, HMQC and $\left.\mathrm{HMBC}\right)$ revealed features characteristic of a triterpene unit containing a 7-membered lactone ring (IR, $1730 \mathrm{~cm}^{-1}$ ) as compared with related compounds isolated from other Cleome species [20, 21]. The ${ }^{1} \mathrm{H}-\mathrm{NMR}$ spectrum of 2 showed seven methyl singlet at $\delta_{\mathrm{H}}$ $1.15,1.26,1.41,1.47,1.53,1.56$, and 2.18 ; which directly correlated with ${ }^{13} \mathrm{C}-\mathrm{NMR}$ signals at $\delta_{\mathrm{c}} 18.3$, $29.2,27.2,23.1,25.9,16.2$, and 31.5 , respectively, an olefinic methyl $\left(\delta_{\mathrm{H}} 2.1\right)$, nine methylenes [including one attached to an acetyl group $\left(\delta_{\mathrm{H}} 4.56 d\right.$ and $\left.4.92 d, \delta_{\mathrm{c}} 61.8\right)$ ], six methines [including one 
oxymethine at $\delta_{\mathrm{c}} 72.9\left(\delta_{\mathrm{H}} 5.15 d\right)$ and two olefinic methines at $\delta_{\mathrm{c}} 126.8\left(\delta_{\mathrm{H}} 4.92 t\right)$ and $128.2\left(\delta_{\mathrm{H}} 5.22\right.$ $d)$ ], and ten quaternary carbons [including three carbonyl carbons $\left(\delta_{\mathrm{c}} 177.6,171.8\right.$ and 171.0), two olefinic carbons $\left(\delta_{\mathrm{c}} 135.0\right.$ and 136.1), and a carbon bearing $\mathrm{OH}$ group at $\left.\left(\delta_{\mathrm{c}} 69.8\right)\right]$. The spectra also revealed the presence of two acetyl groups $\left(\delta_{\mathrm{H}} 2.0 / \delta_{\mathrm{c}} 22.8\right.$ and 2.03/ $\left.\delta_{\mathrm{c}} 21.2\right)$. HMBC correlations of 2 (Figure1) confirmed the gross structure of $\mathbf{2}$ to be a diacetyl triterpene lactone. The relative stereochemistry at C-7 was confirmed to be $7 \beta-\mathrm{H}\left[7 \alpha-\mathrm{H}\right.$ should appear as singlet or $b r s$ near $\delta_{\mathrm{H}} 4.7$ and H-6 should appear as $d(J=1.5 \mathrm{~Hz})$ near $\delta_{\mathrm{H}} 5.9$ as confirmed by ROESY [25], while $\mathrm{H}-7$ appears at $\delta_{\mathrm{H}} 5.15$ as $d(J=10.5 \mathrm{~Hz})$ and $\mathrm{H}-6$ appears at $\delta_{\mathrm{H}} 5.22$ as $\left.d(J=10.5 \mathrm{~Hz})\right]$. The chemical shifts of C-17 and C-21 were found comparable with those of a related compound with $17 \beta-\mathrm{H}\left(\mathrm{C}-17 \delta_{\mathrm{H}} 2.61 d d / \delta_{\mathrm{c}}\right.$ 60.5 and $\left.\mathrm{C}-21 \delta_{\mathrm{H}} 2.24 / \delta_{\mathrm{c}} 31.2\right)$ [26], suggested the $\beta$-orientation of $\mathrm{H}-17$, compared with the data of related compounds with $17 \alpha-\mathrm{H}$ [25-29]. The stereochemistry of the double bond at C-20 (22) was proposed to be a Z-type; since the signal for C-21 was observed at $\delta_{\mathrm{c}} 31.5$ (C-21 of the $E$ - type is usually observed near $\delta_{\mathrm{c}} 13-15$ [25]). From the above data, the structure of 2 was inferred to be as proposed and it was given the name drosericarpone. This compound is reported here for the first time from family Cleomaceae and from nature.

Compound 3, $\mathrm{C}_{35} \mathrm{H}_{58} \mathrm{O}_{6}$, was obtained as fine colorless needles (ethyl acetate), mp, $284^{\circ} \mathrm{C}$, API-MS (positive ion mode) $m / z 613.5\left[(\mathrm{M}+\mathrm{H})^{+} \mathrm{Na}\right]^{+}$. Its structure was identified as stigmasterol glucoside from comparison of its spectral data, $\left({ }^{1} \mathrm{H}\right.$ - and $\left.{ }^{13} \mathrm{C}-\mathrm{NMR}\right)$ with those previously reported [30].

\section{Acknowledgments}

I am grateful to Prof. Dr. Meselhy R. Meselhy (Faculty of Pharmacy, Cairo University, Egypt) for NMR measurements and for valuable discussions. Also, to Prof. Dr. Essam Abdel Sattar (Faculty of Pharmacy, Cairo University, Egypt) and Prof. Dr. Ahmed A. Ahmed (Department of Chemistry, ElMinia University, El-Minia, Egypt) for their valuable comments on the spectral data analysis.

\section{Experimental}

\section{General}

M.p. was measured on a Gallekamp melting point apparatus and was uncorrected. $1 \mathrm{D}-{ }^{1} \mathrm{H}-$ $(500 \mathrm{MHz})$ and ${ }^{13} \mathrm{C}-(125 \mathrm{MHz}) \mathrm{NMR}$ spectra were recorded at $25^{\circ} \mathrm{C}$ using (benzene- $\left.d_{6}\right)$ as solvent and TMS as internal standard on a JEOL 500 Spectrophotometer. 2D-NMR spectra were obtained on a Bruker Avance DRX 400 Spectrophotometer. EI-MS was obtained on Shimadzu PQ-5000 (70 eV) and Bruker Autoflex (Bruker Daltonics, Germany) mass spectrometers. Atmospheric pressure ionization mass spectra (API-MS) were recorded using a PE SCIEX API III bimolecular mass analyzer. Silica gel 60 (70-230 mesh) and Silica gel RP-8 (both from Merck) were used for column chromatography and silica gel $60 \mathrm{H}$ was employed for VLC technique. Centrifugal accelerated radial TLC was performed on a Chromatotron, model no.7924 (Harrison Research Inc. Palo, Alto, Calif., USA). TLC were conducted on precoated silica gel $60 \mathrm{~F}_{254}$ plates $(0.25 \mathrm{~mm}$ thickness, Merck), developed with the solvent system $\mathrm{MeOH}-\mathrm{CHCl}_{3}$ (5:95). The TLC plates were visualized by spraying with $p$-anisaldehyde reagent followed by heating at $110^{\circ} \mathrm{C}$. 


\section{Plant material}

Plant material was collected from the Suez-Cairo desert road, Egypt, in March 2002 and was kindly identified by Dr. M. Gebali (Plant Taxonomy and Egyptian Flora Department, National Research Center, Giza, Egypt). A voucher specimen has been deposited in the herbarium of the Faculty of Pharmacy, Cairo University.

\section{Isolation}

The air-dried powdered herb of $C$. droserifolia $(600 \mathrm{~g})$ was extracted with $70 \%$ ethanol. The residue left after distillation of the solvent (75 g), was successively fractionated with hexane, chloroform and methanol. The hexane extract (3.2 g.) was chromatographed on a VLC column (14 cm $\mathrm{L} \times 4 \mathrm{~cm} \mathrm{D})$ of silica gel $\mathrm{H}(50 \mathrm{~g})$, eluted with hexane, $\mathrm{CHCl}_{3}$, EtOAc and $\mathrm{MeOH}$, in increasing proportions untill $5 \% \mathrm{MeOH}-E t O A c$, in fractions, each of $200 \mathrm{~mL}$. Fraction A: $980 \mathrm{mg}$, eluted with $\mathrm{CHCl}_{3}$, showed a major violet spot with the spray reagent, $\mathrm{R}_{\mathrm{f}}=0.75$. This fraction was further purified by $\mathrm{CC}(18 \mathrm{~cm} \mathrm{~L} \times 3 \mathrm{~cm} \mathrm{D})$ on silica 60 eluted with $5 \% \mathrm{MeOH}_{-} \mathrm{CHCl}_{3}$, in fractions, each of $5 \mathrm{~mL}$, which gave: Fraction $A-1$ (combined frs.7-12, $760 \mathrm{mg}$ ) was further purified twice on a Chromatotron, eluted with $4 \% \mathrm{MeOH} / \mathrm{CHCl}_{3}$, in fractions of $2 \mathrm{~mL}$ each, to give compound 1 (50 mg, oily residue, $\mathrm{R}_{\mathrm{f}}$ $=0.35$ ). Fraction $A-2$ (frs. 40-66, $50 \mathrm{mg}$ ) was further purified twice on a $\mathrm{SiO}_{2} \mathrm{CC}$, eluted with benzene, $\mathrm{CHCl}_{3} \rightarrow 20 \% \mathrm{MeOH}-\mathrm{CHCl}_{3}$, to gave a fraction, which was purified on CC/RP-18 (eluted with water/ $\mathrm{MeOH})$ to give compound $2\left(10 \mathrm{mg}\right.$, oily residue, $\left.\mathrm{R}_{\mathrm{f}}=0.52\right)$. Fraction $B$ (290 mg, eluted with EtOAc) was further purified on a Chromatotron, eluted with $8 \% \mathrm{MeOH}-\mathrm{CHCl}_{3}$, in fractions, each of $2 \mathrm{~mL}$, to give compound 3 (14 mg, fine colorless needles, $\left.\mathrm{R}_{\mathrm{f}}=0.6\right)$.

Buchariol (1), oily residue, $\mathrm{C}_{15} \mathrm{H}_{26} \mathrm{O}_{2}$, [24]; EI -MS (m/z ): 238 ( $\mathrm{M}^{+}$, base peak), 220 ( $\left.\mathrm{M}^{+}-18\right), 195$ $\left(\mathrm{M}^{+}-43, \mathrm{C}_{3} \mathrm{H}_{7}\right), 177\left(\mathrm{M}^{+}-\mathrm{H}_{2} \mathrm{O}-\mathrm{C}_{3} \mathrm{H}_{7}\right), 159\left(\mathrm{M}^{+}-2 \mathrm{H}_{2} \mathrm{O}-\mathrm{C}_{3} \mathrm{H}_{7}\right), 141,81 .{ }^{1} \mathrm{H}-$ and ${ }^{13} \mathrm{C}-\mathrm{NMR}$ spectral data $\left(\mathrm{CDCl}_{3}\right)$ see Table 1 .

Drosericarpone (2), oily residue, $\mathrm{C}_{34} \mathrm{H}_{52} \mathrm{O}_{7}$; EI -MS (m/z): $446\left[(\mathrm{M}+\mathrm{H})-\mathrm{C}_{8} \mathrm{H}_{15} \mathrm{O}\right]^{+}, 388[446-$ $\left.\mathrm{Me}_{2} \mathrm{CO}\right]^{+}, 328$ (388- $\left.\mathrm{CH}_{3} \mathrm{COOH}\right), 286,268$ (388 - $\left.2 \mathrm{x} \mathrm{CH}_{3} \mathrm{COOH}\right), 225,135,127,121$; IR $v_{\max }(\mathrm{KBr})$ $\mathrm{cm}^{-1}: 3440(\mathrm{OH}), 1720,1730$ (carbonyl); ${ }^{1} \mathrm{H}-$ and ${ }^{13} \mathrm{C}-\mathrm{NMR}$ spectral data, see Table 1.

Stigmasterol glucoside (3), fine colorless needles (from ethyl acetate), mp. $284{ }^{\circ} \mathrm{C} ; \mathrm{C}_{35} \mathrm{H}_{58} \mathrm{O}_{6}$; APIMS (positive ion mode) $m / z 613.5\{(\mathrm{M}+\mathrm{H})+\mathrm{Na}\}^{+}, 569.5\left\{(\mathrm{M}+\mathrm{H})+\mathrm{Na}-\mathrm{C}_{3} \mathrm{H}_{8}, 44\right\}^{+}, 525.5$ $\left\{(\mathrm{M}+\mathrm{H})+\mathrm{Na}-2 \mathrm{C}_{3} \mathrm{H}_{8}\right\}^{+}, 481.5\left\{(\mathrm{M}+\mathrm{H})+\mathrm{Na}-3 \mathrm{C}_{3} \mathrm{H}_{8}\right\}^{+}, 413.5\{(\mathrm{M}+\mathrm{H})-162\}^{+}, 393.5$ (base peak ), 349.5, 243, 295.5, 245.5, 133; IR $v_{\max }(\mathrm{KBr}) \mathrm{cm}^{-1}: 3400(\mathrm{OH}), 2960-2850,1640,1465,1380$; ${ }^{1} \mathrm{H}-$ NMR (500 MHz, $\left.C_{6} D_{6}\right): \delta 5.35(1 \mathrm{H}, t, J=4.7 \& 1.7 \mathrm{~Hz}, \mathrm{H}-6), 5.21(1 \mathrm{H}, d d, J=15.2 \& 8.8 \mathrm{~Hz}, \mathrm{H}-22)$, $5.05(1 \mathrm{H}, d d, J=15.2 \& 8.8 \mathrm{~Hz}, \mathrm{H}-23), 4.89\left(1 \mathrm{H}, d, J=7.9 \mathrm{~Hz}, \mathrm{H}-1{ }^{`}\right), 4.42(1 \mathrm{H}, d d, J=2.4 \& 11.7$ Hz, H-6 a $), 4.23\left(1 \mathrm{H}, d d, J=5.3 \& 11.7 \mathrm{~Hz}, \mathrm{H}^{\prime} 6_{\mathrm{b}}^{\prime}\right), 4.12\left(1 \mathrm{H}, m, \mathrm{H}-3{ }^{`}\right), 4.08\left(1 \mathrm{H}, m, \mathrm{H}-4^{\prime}\right) 3.89$ $\left(1 \mathrm{H}, t, J=7.9 \& 8.8 \mathrm{~Hz}, \mathrm{H}-2^{`}\right), 3.84\left(1 \mathrm{H}, m, \mathrm{H}-5^{`}\right), 2.62(1 \mathrm{H}, d d, J=2.6 \& 12.3 \mathrm{~Hz}), 2.37(1 \mathrm{H}, t, J=$ $\left.11.3 \& 11.5 \mathrm{~Hz}, \mathrm{H}-7_{\mathrm{a}}\right), 2.0(1 \mathrm{H}, m, \mathrm{H}-8), 1.9\left(1 \mathrm{H}, m, \mathrm{H}-7_{\mathrm{b}}\right), 0.98(3 \mathrm{H}, d, J=6.4 \mathrm{~Hz}, \mathrm{Me}-21), 0.92$ $(3 \mathrm{H}, s, \mathrm{Me}-19), 0.88(3 \mathrm{H}, d, J=6.8 \mathrm{~Hz}, \mathrm{Me}-26), 0.86(3 \mathrm{H}, d, J=6.8 \mathrm{~Hz}, \mathrm{Me}-27), 0.81(3 \mathrm{H}, t, J=7$ $\mathrm{Hz}, \mathrm{Me}-29), 0.67$ (3H, $s, \mathrm{Me}-18) ;{ }^{13} \mathrm{C}-\mathrm{NMR}\left(125 \mathrm{MHz}, C_{6} D_{6}\right): \delta_{\mathrm{c}} 37.4(\mathrm{C}-1, t) 28.5(\mathrm{C}-2, t), 78.1(\mathrm{C}-$ 
3, d), $39.2(\mathrm{C}-4, t), 140.8(\mathrm{C}-5, s), 121.9(\mathrm{C}-6, d), 32.1(\mathrm{C}-7, t), 32.0(\mathrm{C}-8, d), 50.3(\mathrm{C}-9, d), 36.9(\mathrm{C}-$ 10, s), $21.2(\mathrm{C}-11, t), 39.9(\mathrm{C}-12, t), 42.5(\mathrm{C}-13, s), 56.8(\mathrm{C}-14, d), 24.5(\mathrm{C}-15, t), 29.4(\mathrm{C}-16, t), 56.2$ $(\mathrm{C}-17, d), 12.0(\mathrm{C}-18, q), 19.4(\mathrm{C}-19, q), 36.4(\mathrm{C}-20, d), 19.0(\mathrm{C}-21, q), 137.3(\mathrm{C}-22, d), 128.3(\mathrm{C}-23$, d), $46.0(\mathrm{C}-24, d), 26.3(\mathrm{C}-25, d), 20.0(\mathrm{C}-26, q), 19.2(\mathrm{C}-27, q), 29.9(\mathrm{C}-28, t), 12.2(\mathrm{C}-29, q)$, sugar carbons, $102.4\left(\mathrm{C}-1^{`}, d\right), 75.0\left(\mathrm{C}-2^{`}, d\right), 78.2\left(\mathrm{C}-3^{`}, d\right), 71.5\left(\mathrm{C}-4^{\prime}, d\right), 78.1\left(\mathrm{C}-5^{\circ}, d\right), 62.7\left(\mathrm{C}-6^{\prime}, t\right)$.

Table 1. ${ }^{1} \mathrm{H}$ - and ${ }^{13} \mathrm{C}$-NMR data of compound $\mathbf{1}$ and $\mathbf{2}$

\begin{tabular}{|c|c|c|c|c|c|c|c|c|}
\hline \multicolumn{3}{|c|}{ Compound 1} & \multicolumn{6}{|c|}{ Compound 2} \\
\hline $\begin{array}{c}\text { Position } \\
\text { No. }\end{array}$ & $\delta_{c}$ & $\delta_{H}$ & $\begin{array}{c}\text { Position } \\
\text { No. }\end{array}$ & $\boldsymbol{\delta}_{\mathrm{c}}$ & $\delta_{H}$ & $\begin{array}{c}\text { Position } \\
\text { No. }\end{array}$ & $\boldsymbol{\delta}_{\mathrm{c}}$ & $\boldsymbol{\delta}_{\mathrm{H}}$ \\
\hline 1 & $53.3 d$ & $2.34 m$ & 1 & $34.6 t$ & $\begin{array}{l}\mathbf{2 . 0 5} m \\
\mathbf{2 . 4 6} m\end{array}$ & 16 & $29.3 t$ & $\begin{array}{r}1.36 m \\
1.65 m\end{array}$ \\
\hline 2 & $23.8 t$ & $\begin{array}{l}1.52 m \\
1.57 m\end{array}$ & 2 & $24.6 t$ & $2.26 m$ & 17 & $53.7 d$ & $2.63 m$ \\
\hline 3 & $37.5 t$ & $\begin{array}{l}1.39 m \\
1.75 m\end{array}$ & 3 & $177.6 s$ & - & 18 & $18.3 q$ & $1.15 s 3 \mathrm{H}$ \\
\hline 4 & $74.3 s$ & -- & 4 & $84.8 s$ & - & 19 & 61.8 & $\begin{array}{c}4.56 d \\
4.92 d \\
(12.5 \mathrm{~Hz})\end{array}$ \\
\hline 5 & $68.1 d$ & $2.33 m$ & 5 & $136.1 s$ & - & 20 & $135.0 s$ & - \\
\hline 6 & $75.9 d$ & $\begin{array}{c}4.00(1 \mathrm{H}, \mathrm{br} \\
d d(1.5 \mathrm{~Hz})\end{array}$ & 6 & $128.2 d$ & $\begin{array}{c}\mathbf{5 . 2 2} d \\
(10.5 \mathrm{~Hz})\end{array}$ & 21 & $31.5 q$ & $2.18 s 3 \mathrm{H}$ \\
\hline 7 & $38.5 t$ & $1.38 m$ & 7 & $72.9 d$ & $\begin{array}{c}\mathbf{5 . 1 5} d \\
(10.5 \mathrm{~Hz})\end{array}$ & 22 & $126.8 d$ & $4.92 t$ \\
\hline 8 & $20.2 t$ & $\begin{array}{l}1.55 m \\
1.80 m\end{array}$ & 8 & $49.7 d$ & - & 23 & $44.6 t$ & $\begin{array}{c}2.67 m \\
2 \mathrm{H}\end{array}$ \\
\hline 9 & $48.1 t$ & $\begin{array}{l}2.12 m \\
2.21 m\end{array}$ & 9 & $40.4 d$ & $1.30 \mathrm{~m}$ & 24 & $44.6 t$ & $\begin{array}{c}2.67 m \\
2 \mathrm{H}\end{array}$ \\
\hline 10 & $74.4 s$ & -- & 10 & $45.0 s$ & -- & 25 & $69.8 s$ & - \\
\hline 11 & $32.6 d$ & $1.72 m$ & 11 & $37.2 t$ & $\begin{array}{l}1.28 m \\
1.74 m\end{array}$ & 26 & $29.2 q$ & $1.26 s 3 \mathrm{H}$ \\
\hline 12 & $21.0 q$ & $\begin{array}{c}\mathbf{0 . 9 5} 3 \mathrm{H} d \\
(6.8 \mathrm{~Hz})\end{array}$ & 12 & $26.1 t$ & $\begin{array}{l}1.37 m \\
1.54 m\end{array}$ & 27 & $27.2 q$ & $1.41 s 3 \mathrm{H}$ \\
\hline 13 & $21.0 q$ & $\begin{array}{c}\mathbf{0 . 9 4 3 H ~} d \\
(6.8 \mathrm{~Hz})\end{array}$ & 13 & $40.4 d$ & $1.68 m$ & 28 & $23.1 q$ & $1.47 s 3 \mathrm{H}$ \\
\hline 14 & $25.7 q$ & $1.42 s 3 \mathrm{H}$ & 14 & $49.7 s$ & -- & 29 & $25.9 q$ & $1.53 s 3 \mathrm{H}$ \\
\hline 15 & $21.9 q$ & $\mathbf{1 . 1 8} s 3 \mathrm{H}$ & 15 & $37.8 t$ & $\begin{array}{l}2.08 m \\
2.23 m\end{array}$ & 30 & $16.2 q$ & $1.56 s 3 \mathrm{H}$ \\
\hline
\end{tabular}


Table 1. Cont.

\begin{tabular}{|c|c|c|c|cc|c|cc|}
\hline \multicolumn{3}{|c||}{ Compound 1 } & \multicolumn{6}{c|}{ Compound 2 } \\
\hline $\begin{array}{c}\text { Position } \\
\text { No. }\end{array}$ & $\boldsymbol{\delta}_{\mathbf{c}}$ & $\boldsymbol{\delta}_{\mathbf{H}}$ & $\begin{array}{c}\text { Position } \\
\text { No. }\end{array}$ & $\boldsymbol{\delta}_{\mathbf{c}}$ & $\boldsymbol{\delta}_{\mathbf{H}}$ & $\begin{array}{c}\text { Position } \\
\text { No. }\end{array}$ & $\boldsymbol{\delta}_{\mathbf{c}}$ & $\boldsymbol{\delta}_{\mathbf{H}}$ \\
\cline { 2 - 8 } & & Acetyl & & & Acetyl & & \\
\hline & & at C-7 & $\mathbf{1 7 1 . 0} s$ & - & at C-19 & $\mathbf{1 7 1 . 8} s$ & - \\
& & $22.8 q$ & $\mathbf{2 . 0 0} s 3 \mathrm{H}$ & & $\mathbf{2 1 . 2} q$ & $\mathbf{2 . 0 3} s$ \\
& & & & & & $3 \mathrm{H}$ \\
\hline
\end{tabular}

Assignments were verified using the 2D-NMR $\left({ }^{1} \mathrm{H}-{ }^{1} \mathrm{H}\right.$ COSY, HMQC and HMBC) experiments, and multiplicity was determined by DEPT experiments and $J$ values are given in parenthesis.

\section{References}

1. Täckholm,V. Students `Flora of Egypt, 2nd Ed.; Cairo University Press: Cairo, Egypt, 1974; p. 167.

2. Zohary, M. Flora Palaestina; The Israel Academy of Sciences and Humanities: Jerusalem, 1966; vol. I, p. 245.

3. Boulos, L. Medicinal Plants of North Africa; Reference Publication Inc.: Michigan, USA, 1983; p. 52 .

4. Boulos, L. Flora of Egypt; Al Hadara Publishing: Cairo, Egypt, 1999; p. 177.

5. Ghazanfar, S.A. Handbook of Arabian Medicinal Plants; CRC Press: Boca Raton-London-Tokyo, 1994; p. 76.

6. Chopra, R.N.; Chopra, I.C.; Handa, K.L.; Kapur, L.D. Chopra `s Indigenous Drugs of India; Dhur \& Sons, Private Limited: Calcutta, India, 1972; p. 321, 669.

7. Yang, S.S.; Mabry, T.J.; El-Fishawy, A.M.; El-Kashoury, E.A.; Abdel-Kawy M.A.; Soliman, F.M. Flavonoids of Cleome droserifolia (Forssk.) Del. Egypt. J. Pharm. Sci. 1990, 31, 443.

8. Abdel-Hady, N.M. Pharmacognostical Investigation and Biological Verification of Some Recipes and Preparations of Natural Origin for the Treatment of Diabetes. MS Thesis, Faculty of Pharmacy (Girls), Al-Azhar University, Cairo, 1998.

9. Yaniv, Z.; Dafni, A.; Friedman, J.; Palevitch, D. Plants used for the treatment of Diabetes in Israel. J. Ethnopharmacol. 1987, 19, 145.

10. Nocola, W.G.; Ibrahim, K.M.; Mikhail, T.H.; Girgis, R.B.; Khadr, M.E. Role of the hypoglycemic plant extract Cleome droserifolia in improving glucose and lipid metabolism and its relation to insulin resistance in fatty liver. Boll. Chim. Farm. 1996, 135, 507.

11. Seif El-Din, A.A.; Darwish, F.A.; Abou-Donia, A.A. Flavonoids from Cleome droserifolia (Forssk.) Del. Growing in Egypt. Egypt. J. Pharm. Sci. 1987, 28, 313.

12. Seif El-Nasr, M.M.; Youssef, M.M.; Helmy, M. Flavonoids of Cleome droserifolia (Forssk.) Del. Fitoterapia 1984, 55, 231.

13. Abdel-Kawy, M.A.; El-Deib, S.; El-Khyat, Z.; Mikhail, Y.A. Chemical and biological studies of Cleome droserifolia (Forssk.) Del. Part-I. Egypt. J. Biomed. Sci. 2000, 6, 204. 
14. Fushiya, S.; Kishi, Y.; Hattori, K.; Batkhuu, J.; Takano, F.; Singab, A.N.B.; Okuyama, T. Flavonoids from Cleome droserifolia Suppress NO Production in Activated Macrophages in Vitro. Planta Med., 1999, 65, 404.

15. Diab, L. Pharmacognostical Study of Certain Cleome Species Growing in Egypt. MS Thesis, Faculty of Pharmacy (Boys) Al-Azhar University: Cairo, Egypt, 1992.

16. Harraz, F.M.; Ulubelen, A.; Osuz, S.; Tan, N. Dammarane triterpenes from Cleome amblyocarpa. Phytochemistry 1995,39, 175.

17. Nagaya, H.; Tobita, Y.; Nagae, T.; Itokawa, H.; Takeya, K.; Halim, A.F.; Abdel-Halim, O.B. Cytotoxic triterpenes from Cleome africana. Phytochemistry 1997, 44, 1115.

18. Tsichritzis, F.; Abdel-Mogip, M.; Jakupovic, J. Dammarane triterpenes from Cleome africana. Phytochemistry 1993, 33, 423.

19. Ahmad, V.U.; Qazi, S.; Bin Zia, N.; Xu, C.; Clardy, J. Cleocarpone, A triterpenoid from Cleome brachycarpa. Phytochemistry 1990, 29, 670.

20. Ahmad, V.U.; Alvi, K.A. Deacetoxybrachycarpone, A trinortriterpenoid from Cleome brachycarpa. Phytochemistry 1987, 26, 315.

21. Ahmad, V.U.; Alvi, K.A. The Molecular Structure and Absolute Configuration of Brachycarpone, A new trinortriterpenoid dilactone from Cleome brachycarpa. J. Nat. Prod. 1986, 49, 249.

22. Hussein, N.S.; Ahmed, A.A.; Darwish, F.M. Sesquiterpenes from Cleome droserifolia. Pharmazie 1994, 49, 76.

23. Jente, R.; Jakupovic, J.; Olatunji, G.A. A Cembranoid Diterpene from Cleome viscosa. Phytochemistry 1990, 29, 666.

24. Ahmad, VU.; Zahid, M.; Ali, M.S.; Jassbi, A.R.; Abbas, M.; Ali, Z.; Iqbal, M.Z. Bucharioside and buchariol from Salvia bucharica. Phytochemistry 1999, 52, 1319.

25. Dou, D.; Chen, Y.; Liang, L.; Pang, F.; Shimizu, N.; Takeda, T. Six new dammarane-type trirepene saponins from the leaves of Panax ginseng. Chem. Pharm. Bull. 2001, 49, 442.

26. El-Askary, H. Pregnene glycoside and monoterpene derivative from Solenostemma argel Hayne. Bull. Fac. Pharm. Cairo Univ. 2003, 41, 131.

27. Zou, K.; Zhu, S.; Meselhy, R.; Tohda, C.; Cai, S.; Komatsu, K. Dammarane-type saponins from Panax japonicus and their neurite outgrowth activity in SK-N-SH cells. J. Nat. Prod. 2002, 65, 1288.

28. Nakamura, N.; Kojima, S.; Aura Y.; Meselhy, R.; Hattori, M.; Gupta, M.; Correa, M. Dammarane-type triterpenes from Cordia spinescens. Phytochemistry 1997, 46, 1139.

29. Rouf, A.S.; Ozaki, Y.; Rashid, M.A.; Rui, J. Dammarane derivatives from the dried fruits of Forsythia suspensa. Phytochemistry 2001, 56, 815.

30. Goad, L.J.; Akihisa, T. Analysis of Sterols; Blackie Academic \& Professional: Glasgow, UK. 1997; p. 380.

Sample Availability: Contact the author.

(C) 2005 by MDPI (http://www.mdpi.org). Reproduction is permitted for noncommercial purposes. 